\title{
Reconstruction du climat à partir de la composition isotopique de l'oxygène et du carbone des cernes d'arbres
}

\section{Résumé}

Les compositions isotopiques du carbone et de l'oxygène des cernes d'arbres sont des données exceptionnelles pour reconstituer les variations du climat sur les derniers siècles. Ces compositions sont contrôlées par les processus qui assurent les échanges de dioxyde de carbone et d'eau entre l'arbre et son environnement. Suivant le site et l'espèce d'arbre, ces compositions peuvent être corrélées à la température, à la quantité de précipitation, à l'humidité relative ou même au couvert nuageux. Les reconstitutions climatiques dendro-isotopiques longues sont encore rares. Elles constituent une source d'information unique, notamment du fait de leur résolution annuelle et de leur caractère régional, à confronter aux résultats de la modélisation numérique du climat.

\section{Valérie Daux}

Laboratoire des sciences du climat et de l'environnement, CEA/CNRS/UVSQ, L'Orme des Merisiers, 91191 Gif-sur-Yvette

U ne section dans un tronc d'arbre de forêt tempérée montre, la plupart du temps, une alternance de bandes plus claires et plus sombres autour de la circonférence de l'arbre. Une observation plus fine révèle que ces alternances correspondent à des bandes concentriques de cellules larges aux parois minces (le bois initial produit au printemps) et de cellules plus petites à parois épaisses (le bois final produit en été). Chaque couple de bois initial-bois final constitue un cerne annuel. Les incréments de croissance sont produits au niveau d'un tissu, le cambium, situé à la périphérie du tronc (figure 1).

" - Les paramètres du cerne (largeur, densité et composition du bois) dépendent de nombreuses variables (l'espèce d'arbre, son âge, la disponibilité des éléments nutritifs dans le sol, la teneur en $\mathrm{CO}_{2}$ atmosphérique, etc.) et d'un ensemble complexe de facteurs climatiques (l'ensoleillement, les précipitations, la température, la vitesse du vent, l'humidité de l'air et du sol). Du point de vue de la paléoclimatologie, on peut considérer l'arbre comme un filtre qui, par l'intermédiaire de divers processus physiologiques, convertit un signal d'entrée climatique en différents signaux de sortie, les paramètres du cerne, qui sont enregistrés et peuvent être étudiés même des milliers d'années plus tard. La difficulté pour les dendroclimatologues est de parvenir à séparer le signal climatique enregistré dans les cernes du bruit de fond. S'ils s'intéressent à la variation dans le temps de ce signal, ils devront, de surcroît, connaître précisément l'âge de chaque cerne.

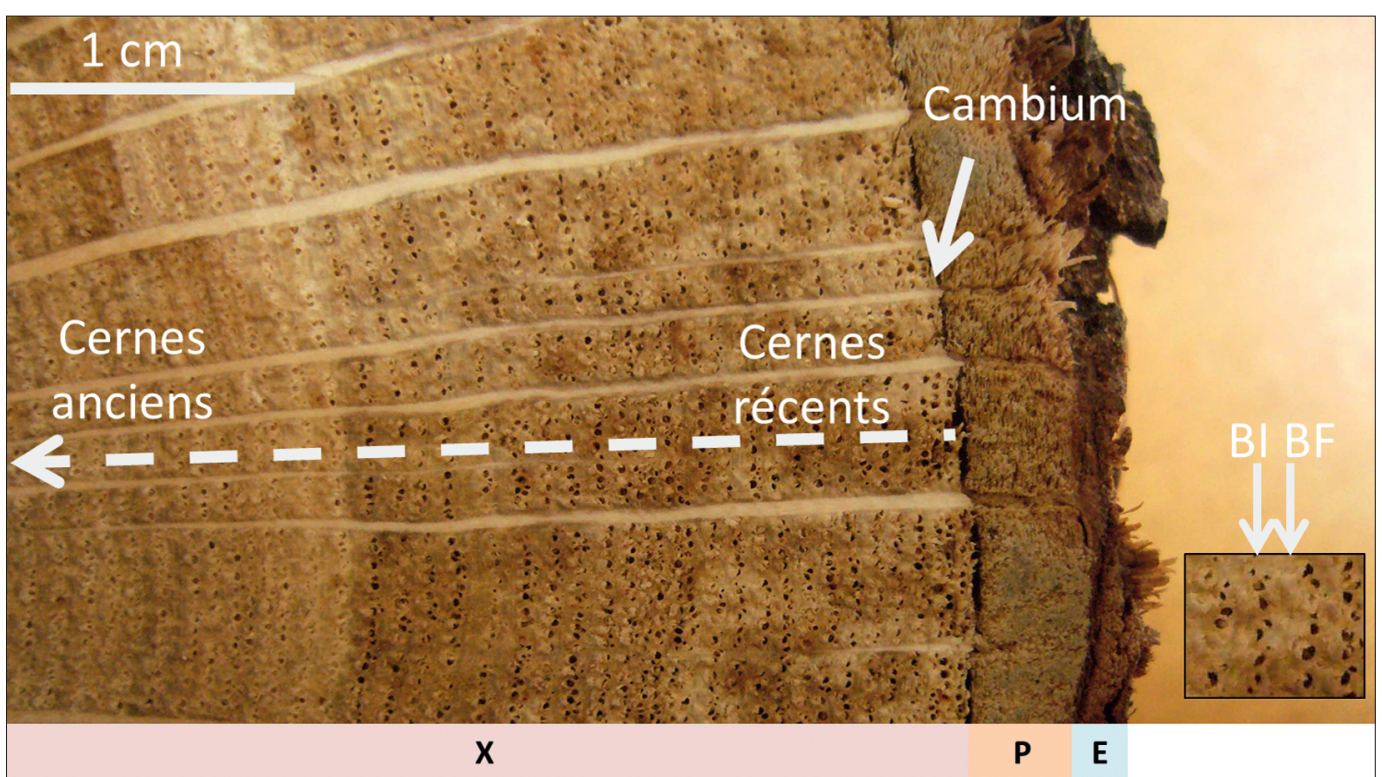




\section{a. . . .}

\section{Abstract}

Reconstructing climate variations from the oxygen and carbon isotopic composition of tree-ring cellulose

Carbon and oxygen isotopic compositions of tree-rings are unrivalled in their potential for paleoclimate reconstruction. These compositions are controlled by the exchanges of $\mathrm{CO}_{2}$ and $\mathrm{H}_{2} \mathrm{O}$ between the tree and its environment. According to the place and the species, $\delta^{13} \mathrm{C}$ and $\delta^{18} \mathrm{O}$ may be correlated with the temperature, the amount of precipitation, the relative humidity or even cloud cover. Long climate reconstructions based on treering stable isotopes are still rare. These regional annually resolved records constitute a unique source of information to be compared with climate model outputs.
L'information climatique a le plus souvent été recherchée dans les variations des largeurs de cernes, mais de nombreuses études ont également porté sur celles de la densité et sont maintenant tournées vers la composition isotopique de la cellulose des cernes.

\section{La dendrochronologie conventionnelle: largeurs de cernes et densité du bois}

Quand les arbres poussent dans des conditions stressantes, en bordure des zones de répartition géographique ou sur un sol pauvre en eau, par exemple, les variations climatiques influencent fortement leur croissance annuelle et ces arbres sont dits « sensibles ». Dans des contextes plus faciles, plus à l'intérieur du domaine écologique ou dans des sites dont l'humidité est optimale pour les arbres, la croissance de ces derniers n'est pas influencée de façon nette par le climat, ce qui se traduit par la faible variabilité interannuelle des largeurs de cernes. De tels arbres sont dits « complaisants ». Il y a ainsi toute une gamme de situations possibles. Dans les études de dendroclimatologie conventionnelle, où les variations de largeur de cernes ou de densité de bois sont la source d'information climatique, on privilégie les arbres qui poussent dans des conditions « stressantes », ce qui conduit souvent à sélectionner des sites en bordure des zones de répartition écologiques (limites arctique ou alpine des arbres par exemple). Des informations climatiques peuvent être obtenues dans des contextes moins stressants pourvu qu'un signal commun à de nombreux échantillons puisse être extrait. Toutefois, à l'échelle mondiale, la répartition des études dendroclimatologiques conventionnelle est biaisée avec une surreprésentation des zones de montagne et de haute latitude (figure 2).

En pratique, l'échantillonnage consiste à prélever des échantillons de bois par carottage. Dans un peuplement donné, le dendrochronologue réalise, en général, 1 à 4 carottes par arbre sur 10 à 20 arbres (figure 3). Chaque carotte est datée par comptage des cernes depuis l'écorce jusqu'au cœur. Chaque cerne est mesuré et un ensemble de 20 à 40 séries temporelles est constitué pour chaque peuplement. En plus de l'épaisseur, il est possible de mesurer la densité grâce à un micro-densitomètre. Ainsi peut-on mesurer l'épaisseur du bois de printemps, celle du bois d'été, la densité moyenne du bois initial et la densité maximale du bois.

Les séries dendrochronologiques sont le plus souvent affectées de tendances liées à l'âge de l'arbre (les cernes deviennent plus minces et le bois moins dense avec l'âge). Des méthodes statistiques sont utilisées pour éliminer le « bruit biologique » des données. Le problème principal de ce processus dit " d'indexation » est qu'il ne distingue pas les tendances biologiques de celles liées à des variations climatiques lentes. En indexant, on risque d'enlever une partie de la tendance liée au climat luimême (Briffa et al., 1996 ; Cook et al., 1995). Depuis quelques années, d'autres méthodes d'indexation sont utilisées (courbe régionale de croissance et méthode par classe d'âge) : elles sont
Figure 2 - Répartition des séries d'indicateurs remontant jusqu'à 1750, utilisées pour reconstruire les variations de température au cours des siècles passés dans le $3^{\mathrm{e}}$ rapport du GIEC (IPCC, 2001). Les triangles marron correspondent aux séries dendroclimatiques. Noter la concentration de ces dernières données dans les zones de haute latitude Nord et dans les zones montagneuses.

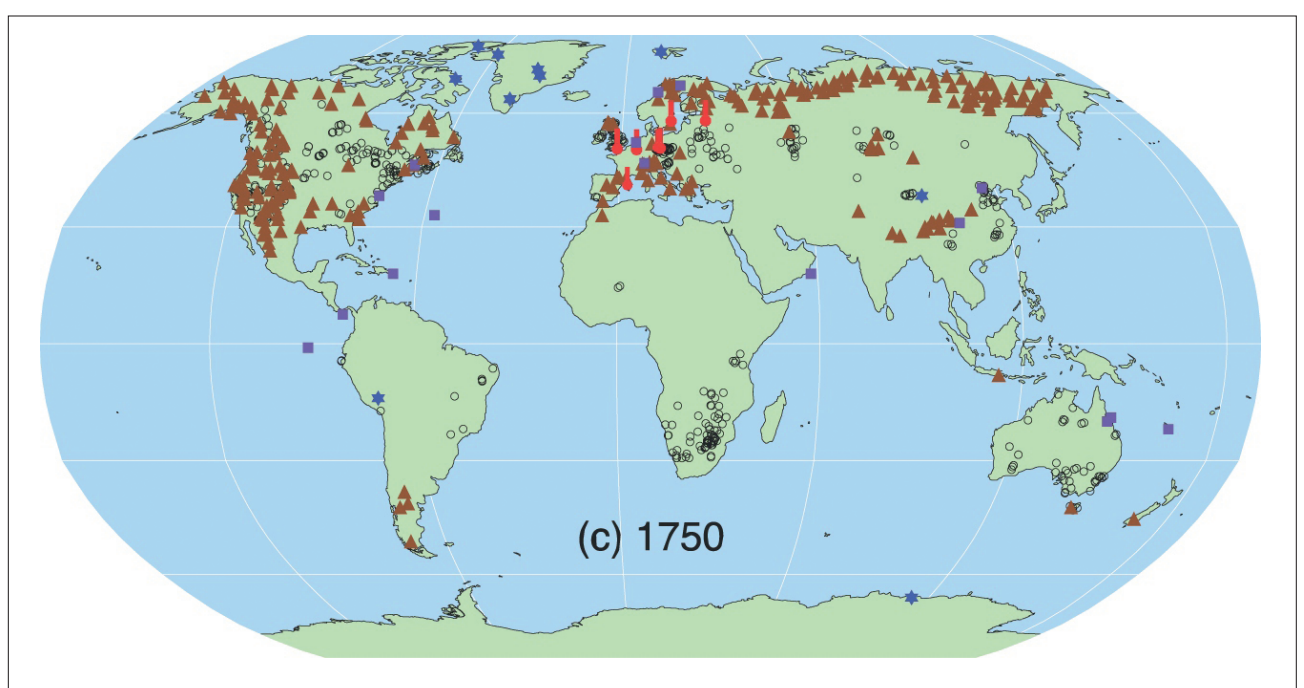



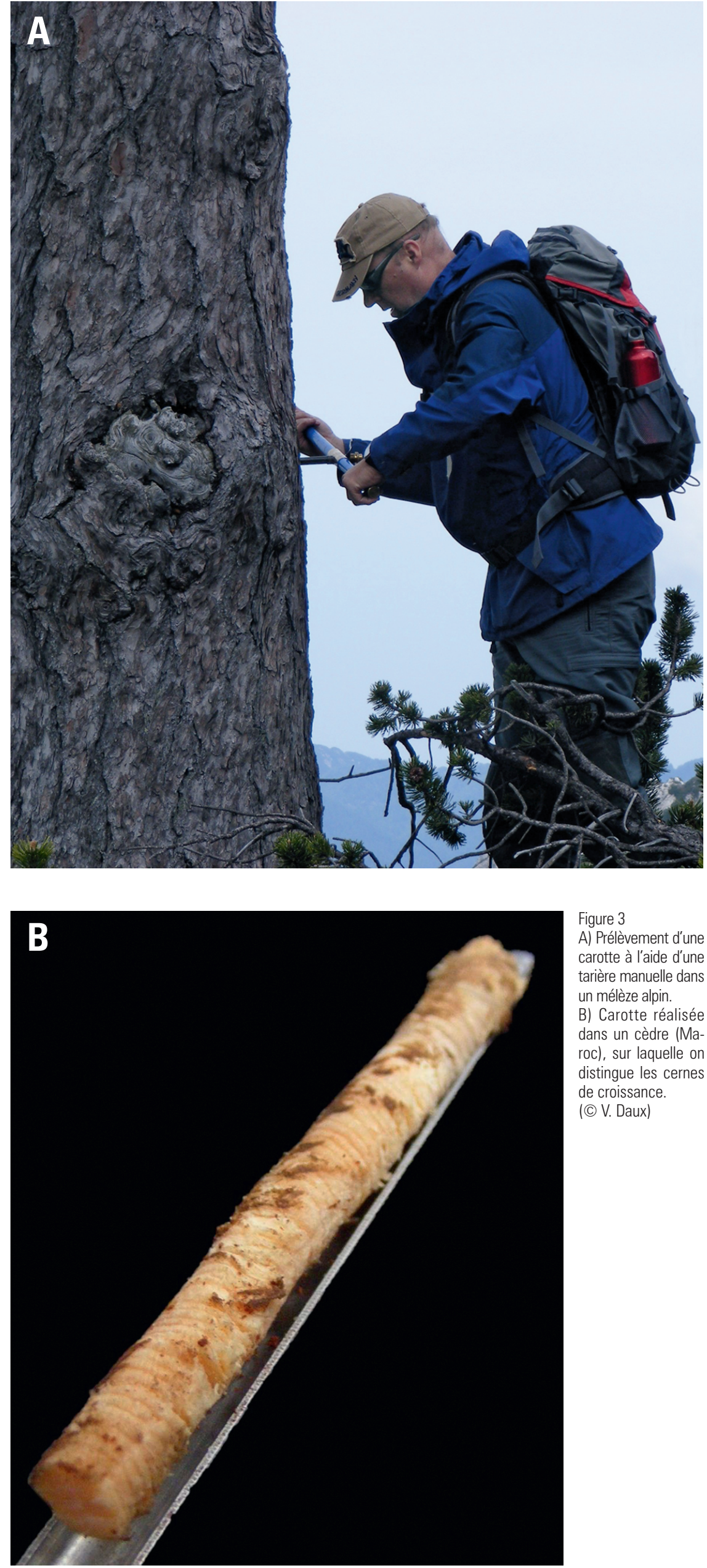
distingue les cernes censées mieux préserver les variations climatiques à long terme (Nicault et al., 2010).

Les séries indexées sont moyennées pour constituer une série représentative du peuplement, appelée chronologie maîtresse. En général, une série maîtresse n'est toutefois pas suffisante pour fournir une reconstruction climatique fiable. Pour une région donnée, on constitue donc un réseau de chronologies maîtresses. Des chronologies longues de plusieurs centaines d'années peuvent être constituées en combinant des séries indexées d'arbres de différents âges (arbres vivants, arbres morts, bois subfossiles et bois de construction).

La reconstruction climatique à partir de ce réseau de chronologies maîtresses s'effectue par une approche statistique qui se décompose en deux étapes : l'étalonnage et la reconstruction stricto sensu. L'étalonnage consiste à déterminer, par des méthodes de régression, les relations qui existent entre les données météorologiques régionales et les paramètres dendrochronologiques, sur la période temporelle commune aux deux types de séries. Le modèle élaboré est vérifié sur un ensemble de données indépendantes. Si le modèle est jugé performant, il peut être utilisé pour effectuer la reconstruction des paramètres climatiques à partir de longues séries de paramètres dendrochronologiques.
Figure 3

A) Prélèvement d'une carotte à l'aide d'une tarière manuelle dans un mélèze alpin.

B) Carotte réalisée dans un cèdre $(\mathrm{Ma}-$ roc), sur laquelle on de croissance. (c) V. Daux)
Les premières reconstructions dendroclimatiques ont été publiées à la fin des années 1970 pour celles basées sur les largeurs de cernes (Blasing et Fritts, 1976 ; Fritts et al., 1979) et vers le milieu des années 1980 pour celles reposant sur les densités du bois (Hughes et al., 1984 ; Schweingrubber et al., 1987).

Pour les études basées sur l'abondance des isotopes stables du carbone, de l'oxygène et de l'hydrogène de la cellulose du bois (dendro-isotopie), la contrainte de sensibilité n'est pas aussi critique qu'en dendrochronologie conventionnelle et des analyses réalisées sur des arbres " complaisants" ont fourni de très bons résultats (par exemple, Etien et al., 2008). Par ailleurs, les compositions isotopiques sont beaucoup moins affectées par des effets d'âge ou de croissance que la densité et les largeurs de cernes. De ce fait, l'approche isotopique est à même de fournir plus d'informations sur la composante basse fréquence des 
variations climatiques (Schweingruber et Briffa, 1996). Enfin, ce ne sont pas nécessairement les mêmes paramètres climatiques qui ont des relations statistiquement significatives avec les largeurs de cernes, la densité et les compositions isotopiques. Ainsi l'approche dendrochronologique conventionnelle et l'approche dendro-isotopique sont-elles complémentaires.

\section{Les isotopes stables}

Les cernes d'arbres sont constitués de matière organique contenant essentiellement du carbone, de l'oxygène et de l'hydrogène. Ces éléments possèdent chacun plusieurs isotopes. L'oxygène, par exemple, a trois isotopes stables ${ }^{16} \mathrm{O}$, ${ }^{17} \mathrm{O}$ et ${ }^{18} \mathrm{O}$, chacun renfermant huit protons mais soit huit, soit neuf, soit dix neutrons. Ces isotopes ont des propriétés chimiques qualitativement identiques mais des masses légèrement différentes. De ce fait, les processus physiques, chimiques ou biologiques peuvent opérer un fractionnement entre les isotopes légers et les isotopes lourds, c'est-à-dire modifier la répartition des isotopes. Les rapports isotopiques de l'oxygène, du carbone et de l'hydrogène portent ainsi une information environnementale. Par convention, le rapport isotopique est exprimé dans une unité relative, le delta $(\delta)$, par référence à un matériau de rapport connu, l'unité étant le pour mil (\%). Pour l'oxygène : $\delta^{18} \mathrm{O}=\left(\mathrm{R}_{\text {ech }} / \mathrm{R}_{\text {ref }}-1\right) \times 1000$, où $\mathrm{R}_{\text {ech }}$ et $\mathrm{R}_{\text {ref }}$ sont respectivement les rapports ${ }^{18} \mathrm{O} /{ }^{16} \mathrm{O}$ de l'échantillon et de la référence. Dans ce cas, la référence est une eau océanique normalisée (Vienna Standard Mean Ocean Water : VSMOW).

Du fait de la différence des masses entre ${ }^{16} \mathrm{O}$ et ${ }^{18} \mathrm{O}$, les molécules d'eau contenant $\mathrm{du}{ }^{16} \mathrm{O}$ s'évaporent plus facilement que celles contenant $\mathrm{du}{ }^{18} \mathrm{O}$. La vapeur est donc isotopiquement plus légère ${ }^{(1)}$ que 1'eau liquide à partir de laquelle elle s'est formée. Les masses d'air froid portent ainsi de la vapeur isotopiquement plus légère que les masses d'air chaud. Quand il s'agit de condensation, on observe le phénomène inverse : les isotopes les plus lourds se condensent plus facilement que les isotopes légers et l'eau de condensation est isotopiquement plus lourde que la vapeur dont elle est issue. La température de la masse d'air détermine la part de vapeur condensable et donc l'amplitude du fractionnement isotopique (phénomène de distillation). Aux moyennes et hautes latitudes, les variations du $\delta^{18} \mathrm{O}$ des précipitations sont liées au premier ordre à la température. Aux basses latitudes, les processus convectifs sont responsables de la majorité des précipitations et les variations du $\delta^{18} \mathrm{O}$ sont principalement liées aux quantités de précipitation (Dansgaard, 1964). Une part des précipitations s'infiltre dans les sols. Dans la partie superficielle de ceux-ci (10 à 20 premiers centimètres), l'évaporation peut entraîner un enrichissement en ${ }^{18} \mathrm{O}$ des eaux d'infiltration par rapport aux précipitations.

L'hydrogène a deux isotopes stables, le plus lourd étant appelé deutérium $\left({ }^{2} \mathrm{H}\right.$ ou D). Le rapport ${ }^{2} \mathrm{H} /{ }^{1} \mathrm{H}$, exprimé en référence au VSMOW comme celui de l'oxygène, s'écrit $\delta \mathrm{D}(\%)$. La vapeur est appauvrie en deutérium par rapport à la source, alors que l'eau de condensation est enrichie par rapport à la vapeur. Comme dans le cas du $\delta^{18} \mathrm{O}$, le $\delta \mathrm{D}$ des précipitations est lié, d'une façon complexe, au climat.

Le carbone a deux isotopes stables, ${ }^{12} \mathrm{C}$ et ${ }^{13} \mathrm{C}$. La référence utilisée pour le calcul du rapport isotopique du carbone dans du bois ou de la cellulose $\left(\delta^{13} \mathrm{C}\right)$, est un fossile de bélemnite de la formation Pee Dee (PDB). D'après cette nomenclature, le $\delta^{13} \mathrm{C}$ du $\mathrm{CO}_{2}$ atmosphérique est actuellement de l'ordre de $-8 \%$. Il a diminué de $1,5 \%$ depuis le début de l'ère industrielle (vers 1850), preuve que l'utilisation de combustibles fossiles a apporté à l'atmosphère des quantités croissantes de $\mathrm{CO}_{2}$ dont le $\delta^{13} \mathrm{C}$ est bas $(\approx-27 \%)$. Le signal enregistré par les arbres reflète l'évolution de la teneur atmosphérique en $\mathrm{CO}_{2}$ et sa composition isotopique. Cette tendance dans les séries de $\delta^{13} \mathrm{C}$ des arbres, qui n'est pas liée au climat, doit être corrigée.

\section{Signature isotopique et physiologie de l'arbre}

Bien que le carbone du bois provienne du $\mathrm{CO}_{2}$ atmosphérique, et que l'oxygène et l'hydrogène viennent de l'eau du sol et donc des précipitations, les rapports isotopiques de ces éléments dans le bois ou la cellulose sont très différents de ceux de l'air ou de l'eau. Les arbres ne sont pas des enregistreurs passifs. Les enregistrements isotopiques dans les cernes d'arbres ne sont pas des échantillons d'air et d'eau

(1) Moins riche en isotopes lourds (Ndlr). du passé mais des bio-indicateurs sensibles qui nous renseignent sur la façon dont les composants de l'eau et de l'air ont été modifiés par l'arbre en réponse aux changements environnementaux.

\section{Oxygène et hydrogène}

Les arbres prélèvent l'eau des sols, par le système racinaire, sans qu'il y ait de fractionnement isotopique (Bariac et al., 1990 ; Wershaw et al., 1966). La composition isotopique de l'oxygène et de l'hydrogène de l'eau du xylème (tissu qui conduit la sève brute ; voir figure 1) est donc la même que celle de l'eau prélevée dans le sol. Bien que les arbres puisent une grande partie de leur eau à des profondeurs situées en dessous de la couche superficielle concernée par les processus d'évaporation (Darling, 2004), on ne peut pas exclure la possibilité que l'eau prélevée soit quelque peu enrichie en isotopes lourds par rapport aux précipitations, au moins à certaines saisons.

D'importants fractionnements se produisent avant que les isotopes ne soient fixés dans les composants du bois. L'étape critique se situe dans les feuilles où l'évapotranspiration entraîne un départ préférentiel de ${ }^{16} \mathrm{O}$ et ${ }^{1} \mathrm{H}$ (Craig et Gordon, 1965 ; Dongmann et al., 1974). Le degré d'enrichissement en isotopes lourds de l'eau de la feuille, qui peut atteindre $20 \%$, dépend de la différence entre la composition isotopique de l'eau du xylème et celle de la vapeur ambiante, ainsi que du rapport des pressions de vapeur à l'intérieur et à l'extérieur de la feuille (figure 4).

Les sucres sont acheminés dans les tissus du phloème (tissus conducteurs de la sève) vers le tronc où ils sont utilisés pour former différentes molécules, notamment de la cellulose. Au cours de la transformation des sucres en cellulose, environ $40 \%$ de l'oxygène et de l'hydrogène sont échangés avec l'eau du xylème (Roden et al., 2000). Ces échanges restaurent, en partie, la signature de l'eau source (figure 4). Dans le cas du deutérium, un enrichissement de l'ordre de $150 \%$ o se produit en plus, au moment où les sucres sont transformés en cellulose (Yakir, 1992). Finalement, le $\delta^{18} \mathrm{O}$ de la cellulose a une valeur variable mais proche de $30 \%$, et le $\delta \mathrm{D}$ a une valeur de l'ordre de $-75 \%$.

Les compositions isotopiques de l'oxygène et du deuterium de la cellulose des cernes d'arbres reflètent celles de la source (l'eau du xylème, c'est-à-dire des précipitations via le sol) et le degré 


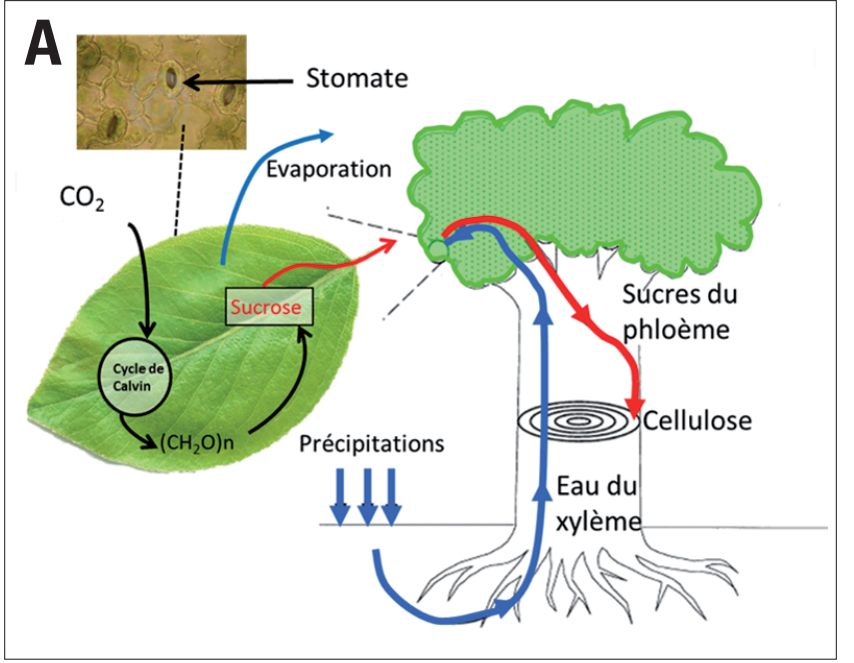

Figure 4

A) Schémas du fonctionnement de l'arbre.

B) Schémas des différents compartiments isotopiques pour l'oxygène. La couleur bleue correspond à l'eau non chargée en sucres (précipitations, eau du xylème et eau évaporée), et la couleur rouge à l'eau chargée en sucres synthétisés dans la feuille.

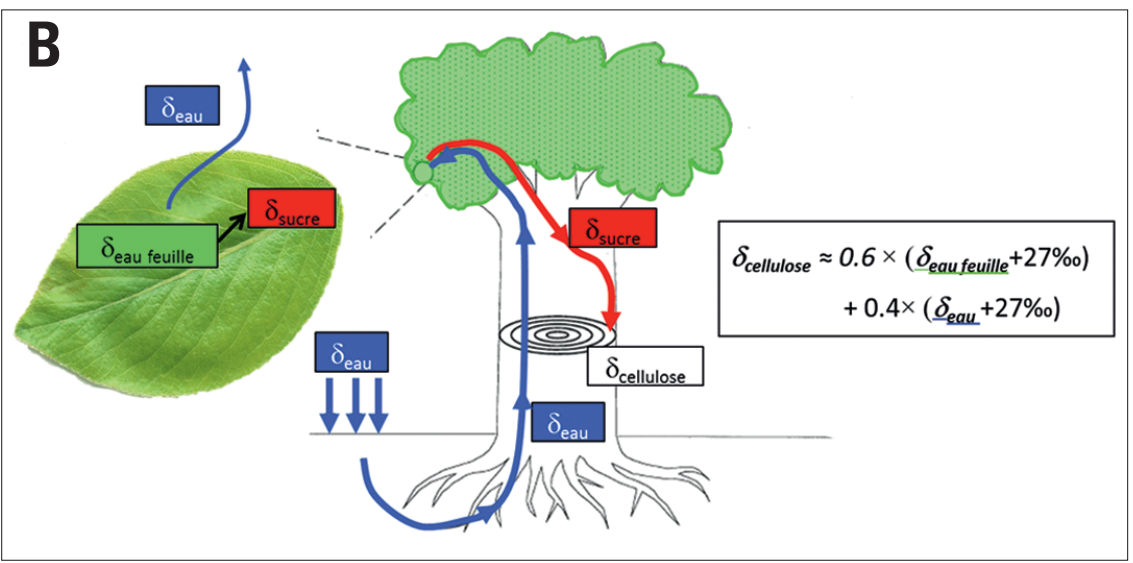

d'enrichissement par évaporation dans la feuille (contrôlé par la température et l'humidité de l'air et du sol). Les variations de $\delta^{18} \mathrm{O}$ et le $\delta \mathrm{D}$ dans la cellulose sont donc liées à celles du climat.

\section{Carbone}

La différence entre le $\delta^{13} \mathrm{C}$ du bois et de la cellulose $(\approx-25 \%)$ et celui de l'atmosphère $(\approx-8 \%$ ), aussi appelée discrimination, est contrôlée par la physiologie de l'arbre et par son environnement (humidité relative, teneur en eau du sol, température, ensoleillement et disponibilité des éléments nutritifs). Les arbres effectuent leurs échanges gazeux avec l'atmosphère (vapeur d'eau de l'intérieur vers l'extérieur, et $\mathrm{CO}_{2}$ dans le sens inverse) par l'intermédiaire de pores, les stomates, situés sur la face inférieure des feuilles, le plus souvent. L'ouverture des stomates est régulée par les cellules de garde dont le fonctionnement doit garantir à l'arbre des entrées de $\mathrm{CO}_{2}$ atmosphérique sans perte excessive d'humidité. Le $\mathrm{CO}_{2}$ est utilisé par des enzymes photosynthétiques qui produisent les sucres. Au cours de la transformation du $\mathrm{CO}_{2}$ atmosphérique en sucre, deux types de fractionnements peuvent se produire. Quand l'air diffuse au travers des stomates, les molécules de $\mathrm{CO}_{2}$ qui renferment les isotopes de carbone les plus légers $\left({ }^{12} \mathrm{C}\right)$ diffusent plus rapidement que celles renfermant les isotopes les plus lourds $\left({ }^{13} \mathrm{C}\right)$. L'air qui se trouve à l'intérieur des feuilles (air interne) est par conséquent appauvri en ${ }^{13} \mathrm{C}$ par rapport à l'air ambiant. Une deuxième étape de fractionnement se produit quand le $\mathrm{CO}_{2}$ interne est utilisé par les enzymes photosynthétiques. $\mathrm{Ce}$ processus favorise $1 \mathrm{le}{ }^{12} \mathrm{C}$ au détriment $\mathrm{du}{ }^{13} \mathrm{C}$. Les sucres produits sont donc appauvris par rapport au $\mathrm{CO}_{2}$ interne. Il a été montré que la discrimination est liée au rapport des pressions partielles interne et externe de $\mathrm{CO}_{2}$ (Farquhar et al., 1982). Comme la pression interne est régulée par l'ouverture des stomates, elle-même contrôlée par l'état hydrique de l'arbre, il existe un lien entre la discrimination du carbone et le climat (température, taux d'humidité et insolation).

Le $\delta^{13} \mathrm{C}$ de la cellulose des cernes d'arbres peut présenter des tendances liées à l'âge, moins marquées et sur un nombre d'années moins important que l'effet qui affecte les largeurs de cernes et la densité du bois. L'effet, dit « juvénile », se caractérise par des valeurs plus basses du $\delta^{13} \mathrm{C}$ de la cellulose du bois produit au cours des premières décennies (Francey et Farquhar, 1982). Dans la plupart des cas, les compositions isotopiques de l'oxygène et de l'hydrogène de la cellulose ne sont pas affectées par des effets juvéniles, ce qui confère à ces paramètres un intérêt particulier pour la reconstitution des variations climatiques à basse fréquence (pluri-décennales à séculaires).

\section{Méthode et procédure analytique}

Les échantillons de bois destinés à l'analyse isotopique sont prélevés par carottage. Comme en dendroclimatologie conventionnelle, pour constituer des séries longues, il est nécessaire d'associer des bois vivants, morts, de construction ou même subfossiles avec des périodes de recouvrement entre les différentes carottes. Par ailleurs, pour une année calendaire donnée, on estime qu'il est nécessaire d'analyser au moins 4 individus distincts pour obtenir un signal représentatif de la population. Une fois la chronologie établie, les carottes sont découpées cerne à cerne, éventuellement en séparant bois initial et bois final. Les cernes sont broyés puis soumis à une attaque chimique qui élimine les composés autres que la cellulose (graisses, résines, lignine, hémicellulose, etc.). Cette étape (figure 5) est nécessaire car ces différents composés ont des compositions isotopiques sensiblement différentes les unes des autres. Aussi les paramètres météorologiques sont-ils liés par des relations plus fortes (car moins bruitées) aux rapports isotopiques de l'oxygène et du carbone de la cellulose qu'à ceux du bois total.

Figure 5 - Illustration du protocole analytique découpe cerne à cerne, purification de la cellulose et analyse au spectromètre de masse.

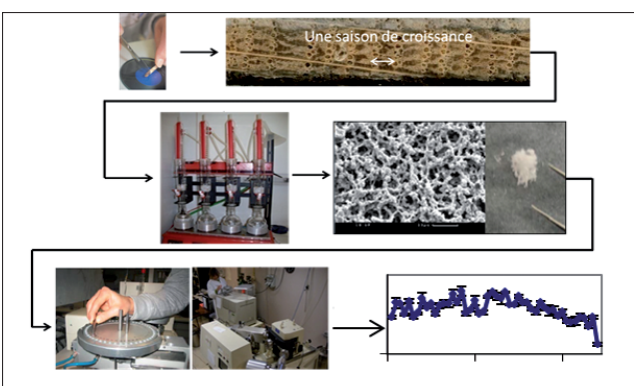


Du fait de la configuration de la molécule de cellulose, les échanges d'atomes d'oxygène et de carbone avec d'autres composés ne sont pas possibles. Ainsi, la cellulose d'un bois immergé pendant une très longue période dans l'eau conservet-elle son $\delta^{18} \mathrm{O}$ et son $\delta^{13} \mathrm{C}$ originels (Savard et al., 2012). En revanche, jusqu'à $30 \%$ des atomes d'hydrogène de ce matériau peuvent être échangés avec des sources externes (avec l'humidité atmosphérique, par exemple). Une étape d'équilibration avec de l'eau de composition connue, ou de nitration (remplacement des $\mathrm{H}$ échangeables par $\mathrm{NO}_{2}$ ), est donc nécessaire avant de procéder à l'analyse isotopique de l'hydrogène.

Pour réaliser l'analyse isotopique d'O, $\mathrm{H}$ et $\mathrm{C}$, il faut que la cellulose soit transformée en une phase gazeuse analysable par spectrométrie de masse. Cette étape est effectuée de plus en plus fréquemment par une combustion ou par une pyrolyse de la matière à haute température $\left(1100^{\circ} \mathrm{C}\right.$ ou plus $)$, dans un four en ligne avec le spectromètre de masse. Le gaz produit $\left(\mathrm{CO}, \mathrm{CO}_{2}\right.$ ou $\left.\mathrm{H}_{2}\right)$ est véhiculé par un gaz vecteur inerte jusqu'au spectromètre où la mesure a lieu. La précision des mesures est de l'ordre de $0,1 \%$ pour $\delta^{13} \mathrm{C}$ et de l'ordre de 0,15 $0,2 \%$ pour $\delta^{18} \mathrm{O}$.

La nécessaire étape d'équilibration avant l'analyse spectrométrique de l'hydrogène rend l'ensemble de l'opération plus difficile et plus hasardeux que dans le cas du carbone et de l'oxygène. Par ailleurs, l'interprétation des variations de $\delta \mathrm{D}$ est souvent complexe et parfois controversée. Pour cette raison, peu d'études portent sur l'utilisation de $\delta \mathrm{D}$ comme indicateur climatique. Seuls des résultats obtenus avec $\delta^{18} \mathrm{O}$ et $\delta^{13} \mathrm{C}$ seront présentés dans la partie qui suit.

\section{Reconstitution des variations climatiques à partir de la compo- sition isotopique de l'oxygène et du carbone de la cellulose}

Les variations de la composition isotopique de l'oxygène et du carbone de la cellulose des arbres sont interprétées dans la littérature de façons très diverses. Le signal paléoclimatique reconstruit peut être la température, l'humidité relative, les quantités de précipitation, la couverture nuageuse, le stress hydrique, l'insolation ou encore le $\delta^{18} \mathrm{O}$ des précipitations. Cette disparité est liée, d'une part, à la variété des espèces et des environnements sur lesquels reposent les reconstructions et, d'autre part, à l'état des connaissances éco-physiologiques. Par exemple, on connaît encore assez mal l'incidence sur les fractionnements isotopiques des interactions complexes et variables entre différents processus physiologiques (par exemple, la mobilisation des réserves versus l'utilisation des sucres de la saison pour la construction des cernes). Pour combler cette lacune, et dans la perspective de comprendre les relations qui lient les paramètres isotopiques de la cellulose aux paramètres climatiques, des modèles couplés sont élaborés. Ces derniers associent des modèles de fractionnement isotopique dans l'arbre à des modèles qui simulent la réponse de la végétation aux changements climatiques. Cette voie de recherche est en cours de développement et elle n'a pas encore permis de réaliser des reconstitutions climatiques à partir de données dendro-isotopiques.

Pour réaliser de telles reconstitutions, la démarche adoptée consiste à traiter le système " arbre » comme une « boîte noire » et à appréhender les relations entre paramètres isotopiques et climatiques sous l'angle statistique. La méthodologie est identique à celle utilisée en dendroclimatologie conventionnelle, à savoir : les relations (aussi appelées fonctions de transfert) qui existent entre les paramètres isotopiques et climatiques sont établies sur la période instrumentale, la qualité des fonctions de transfert est établie sur des données indépendantes de celles utilisées pour la calibration, et, enfin, les fonctions de transfert sont utilisées pour transformer les séries de données dendro-isotopiques en séries climatiques reconstruites.

Les reconstructions climatiques dendroisotopiques sont très nombreuses dans la littérature. La sélection qui est présentée ci-après a été réalisée dans le souci d'illustrer au mieux, en quelques lignes, le potentiel de $\delta^{18} \mathrm{O}$ et de $\delta^{13} \mathrm{C}$ comme indicateurs climatiques, et de donner un aperçu de la variété possible des sites étudiés (montagne/plaine), des espèces d'arbres utilisées (genévrier, pin à crochets et chêne, dans ces exemples), et des paramètres reconstruits (précipitations et température). La longueur exceptionnelle des séries climatiques reconstruites (de 700 à plus de 1000 ans) a également présidé à la sélection des trois exemples qui suivent.

\section{Reconstitution de la quantité de précipitations dans I'Ouest de l'Himalaya à partir du $\delta^{18} 0$ de genévriers}

Les sites étudiés par Treydte et al. (2006) se situent au Pakistan, dans les montagnes du Karakorum, entre

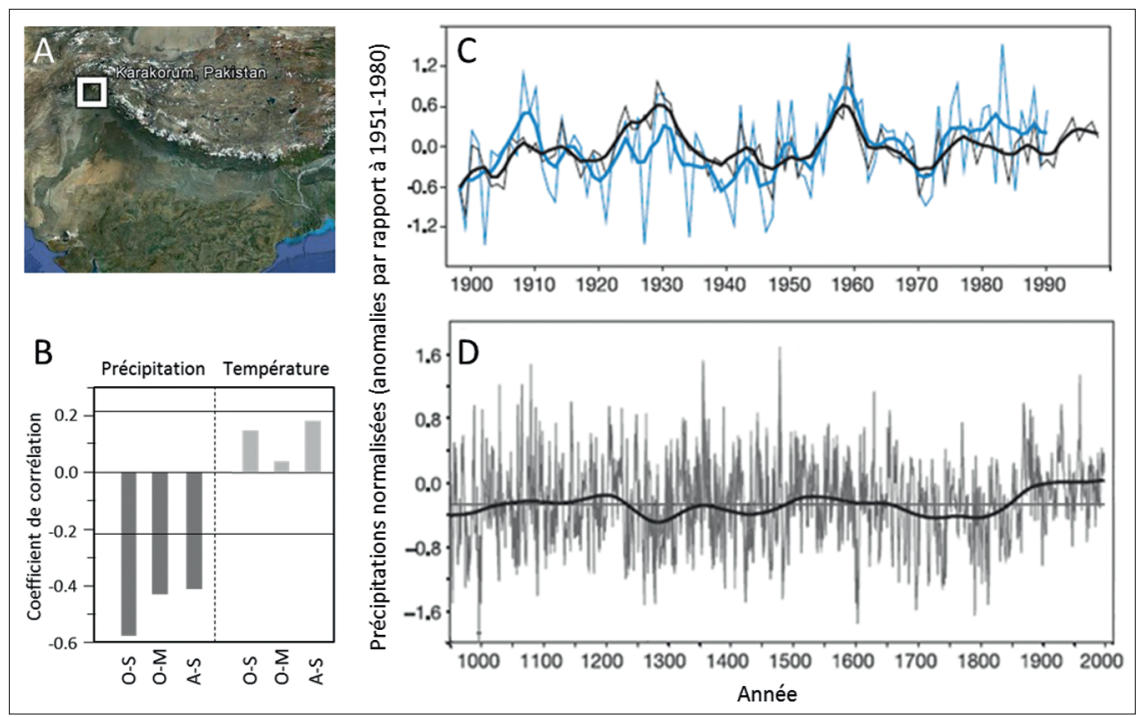

Figure 6 - Reconstitution des variations de précipitations au cours du dernier millénaire dans l'Ouest de l'Himalaya (Treydte et al., 2006).

A) Localisation de la région du Karakorum (Pakistan).

B) Coefficients de corrélation entre la série de $\delta^{18} 0$ de la cellulose de genévriers et les précipitations et températures annuelles (O-S), hivernales (O-M) et estivales (A-S).

C) Comparaison des séries de précipitations normalisées instrumentales (en bleu) et des séries reconstruites à partir des $\delta^{18} \mathrm{O}$ (en noir).

D) Variation des précipitations annuelles au Karakorum au cours du millénaire (trait fin). Les variations à long terme sont soulignées par une courbe spline à 150 ans. 
$2900 \mathrm{~m}$ et $3700 \mathrm{~m}$ (figure 6A). Les arbres analysés sont des genévriers (Juniperus excelsa et $J$. turkestanica). Des corrélations fortes $(\mathrm{R}<-0,58)$ et statistiquement significatives $(\mathrm{p}<0,001)$ ont été mises en évidence entre les précipitations de l'année biologique (octobre à septembre) et le $\delta^{18} \mathrm{O}$ de la cellulose. Des corrélations moins fortes existent avec la température ou les précipitations d'hiver (octobremars) ou de la saison de croissance (avril-septembre; voir figures 6B et ${ }_{6 C)}$. Sur cette base, la série de $\delta^{18} O$ à résolution annuelle, qui a été établie à partir de genévriers millénaires, a été transformée en une chronologie de variations des précipitations au cours du temps. D'après cette reconstitution, des groupes d'années humides ont marqué la fin $\mathrm{du} \mathrm{XIX}^{\mathrm{c}}$ et le $\mathrm{XX}^{\mathrm{e}}$ siècle dans cette région du monde. Les derniers 150 ans constituent ainsi la période la plus humide de tout l'enregistrement. Des périodes humides décennales sont repérables autour de $1200,1350,1500$ et 1870 , alors que des périodes sèches, d'une ou de plusieurs dizaines d'années, sont enregistrées avant l'an 1000 et autour de $1270,1420,1600,1720,1790$ et 1890 . Ces deux dernières décennies sont connues comme des épisodes de famine sévère en Inde. D'après les auteurs, le synchronisme de l'augmentation des précipitations au Pakistan et dans d'autres sites asiatiques, européens et américains pourrait révéler une intensification du cycle hydrologique en coïncidence avec le réchauffement climatique.

จ Figure 7 - Reconstruction des précipitations estivales pour la période 1085-2005 en Californie, États-Unis (Bale et al., 2011).

A) Localisation du site de prélèvement de pins à cônes épineux dans les White Mountains en Californie.

B) Reconstruction à partir du $\delta^{13} \mathrm{C}$ de la cellulose des pins. Les événements El Niño extrêmes sont repérés par des lignes pointillées. Ceux des 500 dernières années (1500-2005) ont été identifiés par Gergis et Fowler (2009) et Quinn et Neal (1992), et ceux des années 1085-2005 par Bale et al. (2011) [années 1087, 1091, 1120, 1121, 1122, 1247, 1266, 1267, 1343, 1349, 1422, 1486 et 1487]. Le nombre d'arbres analysés est représenté par la ligne continue dans la partie inférieure de la figure. Les zones bleutées correspondent à des périodes de sécheresse. Noter l'inversion de l'échelle des précipitations.

\section{Reconstitution des phases El Niño à partir du $\delta^{13} \mathrm{C}$ de pins américains au cours du dernier millénaire}

Une chronologie de $\delta^{13} \mathrm{C}$ de plus de 1000 ans a été établie à partir des analyses de cellulose des pins à cônes épineux (Pinus longaeva, pins bristlecone, D. K. Bailey), d'une extraordinaire longévité, qui se développent dans la région des White Mountains en Californie (Bale et al., 2011 ; figure 7). Une fois corrigés les changements du $\delta^{13} \mathrm{C}$ atmosphérique liés à l'utilisation de combustibles fossiles, les $\delta^{13} \mathrm{C}$ de la cellulose de ces pins apparaissent bien corrélés aux précipitations estivales (juin à août; $\mathrm{R}=-0,5 ; \mathrm{p}<0,05$ ). De plus, les valeurs les plus basses de $\delta^{13} \mathrm{C}$ coïncident avec les événements El Niño les plus sévères des 500 dernières années. En partant de cette constatation et en faisant implicitement l'hypothèse que les téléconnections ENSOprécipitations et précipitations-proxies sont constantes, les auteurs identifient 13 événements El Niño extrêmes possibles au cours des 400 années précédentes. Ces événements seraient concentrés autour de 1100, 1250, 1350 et 1500 .

\section{Reconstitution des températures dans la moitié Nord de la France à partir du $\delta^{18} 0$ de chênes au cours des 700 dernières années}

Une chronologie de $\delta^{18} \mathrm{O}$, remontant jusqu'en 1306, a été constituée à partir de bois d'arbres vivants de la forêt et de poutres du château de Fontainebleau (Etien et al., 2008 ; Etien et al., 2009 et données non publiées). Parmi tous les paramètres météorologiques testés, les températures maximales moyennées sur la période avril-septembre $\left(\mathrm{T}_{\max }{ }^{\text {AMJAS }}\right)$ sont celles avec lesquelles les corrélations sont les plus fortes sur la période instrumentale $\left(\mathrm{R}=0,58 ; \mathrm{p}=6.10^{-12}\right)$ [voir figure 8]. La reconstitution des

จ Figure 8 - Reconstitution des températures dans la moitié Nord de la France à partir du $\delta^{18} 0$ des chênes de Fontainebleau, France (Etien et al., 2008 ; Etien et al., 2009 et données non publiées).

A) Localisation du site d'étude.

B) Comparaison des variations, au cours du temps, des températures ( $T_{\max }$ AMJAs ; voir texte) calculées et mesurées. En rouge : température instrumentale ; en noir : température reconstruite à partir du $\delta^{18} 0$ de la cellulose des chênes de Fontainebleau. Les traits fins correspondent aux variations interannuelles, les traits épais à des moyennes glissantes sur 11 ans. La zone ombrée grise représente la zone d'incertitude calculée pour les températures reconstruites.

C) Reconstitution des variations de température au cours des 7 derniers siècles. (Même légende pour les couleurs et les traits que dans la figure B.)
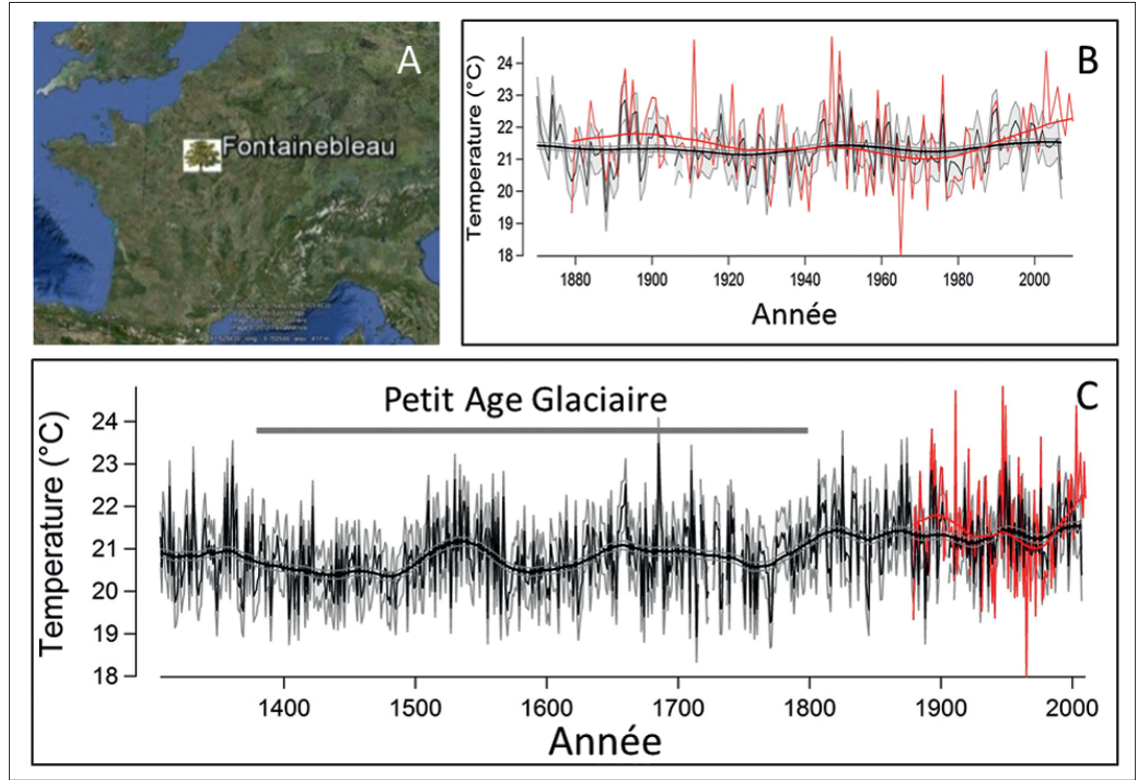
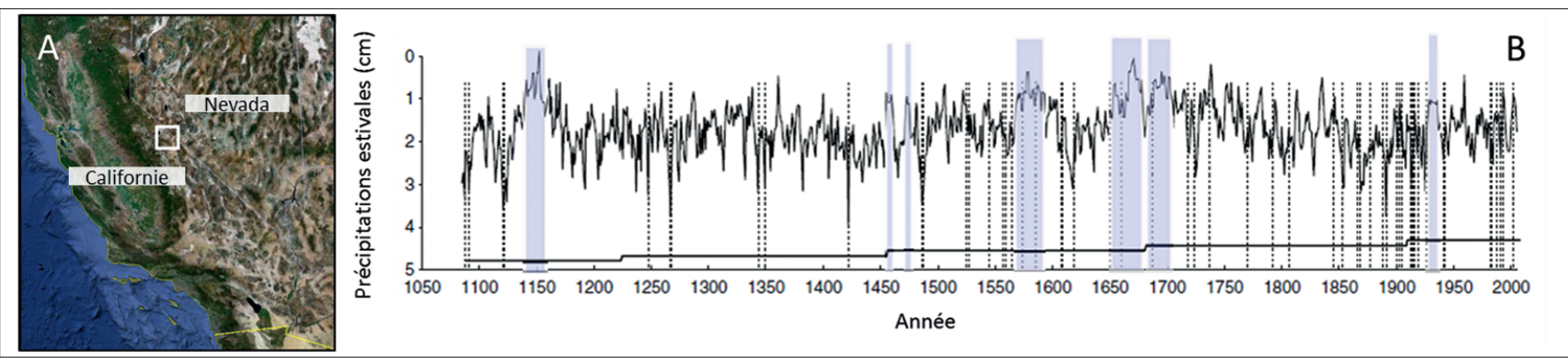
températures sur les 700 dernières années permet de distinguer une période plus froide débutant au XIV ${ }^{\mathrm{e}}$ siècle se terminant au début du XIX ${ }^{\mathrm{e}}$, interrompue par quelques décennies plus clémentes au XVI et à la fin du XVII ${ }^{\mathrm{e}}$ siècle. Les périodes de péjoration du climat coüncident avec le Petit Âge Glaciaire. Les deux dernières décennies présentent une tendance au réchauffement, inégalée par sa durée et son amplitude. La série thermométrique reconstruite présente de nombreuses similitudes avec d'autres séries européennes, ce qui montre qu'elle a une valeur régionale.

Sur la période post-1990, les températures mesurées sont plus élevées que celles calculées à partir de $\delta^{18} \mathrm{O}$, et l'écart entre les deux courbes s'accroît au cours du temps. Parmi les causes invoquées pour expliquer ce type de divergence, observé également en quelques autres sites (par exemple, Hilasvuori et al., 2009 ; Daux et al., 2011), on peut citer les effets possibles de l'assèchement des sols en surface, de la modification de la période de croissance, ou encore de la pollution atmosphérique. Cette divergence est réellement problématique car elle soulève la question de la stabilité des relations entre composition isotopique et climat au cours du temps.

\section{Conclusion}

Les arbres présentent des atouts certains en paléoclimatologie :

1) ils sont répandus sur une grande partie des surfaces continentales;

2) dans les régions à climat méditerranéen, tempéré, subarctique, ou montagneux, les arbres fabriquent des cernes annuels ;
3) en combinant les bois d'arbres vivants, de poutres de bâtiments historiques, voire d'arbres subfossiles, il est possible de réaliser des chronologies de plusieurs centaines d'années, datées avec précision ;

4) l'analyse isotopique de quelques individus suffit pour obtenir un signal à valeur régionale,

5) et, enfin, les variations de la composition isotopique de l'oxygène et du carbone de la cellulose des cernes sont en général fortement corrélées à celles de paramètres climatiques.

Les arbres ne sont néanmoins pas des archives climatiques parfaites :

- les effets de l'âge sur $\delta^{13} \mathrm{C}$ (plus rares sur $\left.\delta^{18} \mathrm{O}\right)$, s'ils restent modérés par rapport à ceux qui affectent la croissance, sont relativement variables d'un site et d'une espèce à l'autre ;

- la diminution progressive du $\delta^{13} \mathrm{C} d u$ $\mathrm{CO}_{2}$ atmosphérique depuis 1850 impose de corriger les séries de $\delta^{13} \mathrm{C}$ des cernes. À cet effet s'ajoutent ceux de l'augmentation du $\mathrm{CO}_{2}$ sur le fonctionnement même des arbres, avec une incidence démontrée sur leur croissance et une incidence possible sur les fractionnements isotopiques dans l'arbre ;

- la divergence entre les séries thermométriques et les séries de température reconstituées à partir des isotopes de la cellulose sur la période post-1990, qui a été mise en évidence dans plusieurs sites, remet en cause la notion de stabilité temporelle des relations entre paramètres isotopiques et climatiques.

Pour surmonter ces écueils, plusieurs voies sont explorées et elles nécessitent la collaboration entre géochimistes, écophysiologistes, historiens et modélisateurs. La première voie consiste à combiner différentes archives (arbres-dates de vendanges, arbres-lacs, arbres-spéléothèmes, arbres-glaciers, etc.), dans l'optique de minimiser les biais propres à chacune (par exemple, Etien et al., 2009 ; Sidorova et al., 2010). La seconde voie, expérimentale, se consacre à la mesure des compositions isotopiques des fluides (pluie, eau du sol et des feuilles, sève et vapeur) et des différents composants de l'arbre (sucre, cellulose, lignine, etc.), y compris avec une résolution infraannuelle. Enfin, dans la dernière voie, modélisatrice, les isotopes de l'oxygène et du carbone sont intégrés dans les modèles numériques de climat (sol, végétation, atmosphère et océan), dans le but de mieux comprendre les liens de causalité entre les changements climatiques et les variations de la composition isotopique de la cellulose. Les progrès réalisés dans le domaine expérimental et dans la modélisation améliorent progressivement notre compréhension des mécanismes et des interactions complexes qui entraînent des fractionnements et des échanges isotopiques dans l'arbre. Ces trois nouvelles approches sont en plein développement. Elles permettront de raffiner l'interprétation des séries dendro-isotopiques et apporteront, en outre, des informations cruciales pour évaluer les échanges d'eau et de carbone entre les surfaces continentales et l'atmosphère.

\section{Remerciements}

Tous mes remerciements à Tom Levanic pour avoir accepté que son image soit utilisée pour illustrer le carottage des arbres, à Monique Pierre et à Michel Stievenard pour les échanges quotidiens stimulants et la relecture de cet article, ainsi qu'à Valérie Masson-Delmotte et à un rapporteur anonyme pour leurs suggestions pertinentes pour améliorer le manuscrit initial.

\section{Bibliographie}

\footnotetext{
: Bale R. J., I. Robertson, M. W. Salzer, N. J. Loader, S. W. Leavitt, M. Gagen, T. P. Harlan et D. McCarroll, 2011 : An annually resolved bristlecone pine carbon isotope chronology for the last millenium. Quat. Res., 76, 22-29.

- Bariac T., C. Jusserand et A. Mariotti, 1990 : Évolution spatio-temporelle de la composition isotopique de l'eau dans le continuum sol-plante-atmosphere. Geochim. - Cosmochim. Acta, 54, 413-424

- Blasing T. J. et H. C. Fritts, 1976 : Reconstructing past climate anomalies in the North Pacific and western North America from tree-ring. Ouat. Res., 6, 563-579.

- Briffa K. R., P. D. Jones, F. H. Schweingrubber, W. Karlen et S. G. Shiyatov, 1996 : Tree-ring variables as proxy-climate indicators: Problems with low frequency

- signals. Dans : Climate variations and forcing mechanisms of the last 2000 years, P. D. Jones, R. S. Bradley et J. Jouzel, Eds., Springer-Verlag, New York, 9-41.
} 
: Cook E. R., K. R. Briffa, D. M. Meko, D. S. Graybill et G. Funkhouser, 1995 : The 'segment length curse' in long tree-ring chronology development for paleoclimatic studies. The Holocene, 5, 229-237.

- Craig H. et L. I. Gordon, 1965 : Deuterium and oxygen 18 variations in the ocean and the marine atmosphere. Dans : Stable isotopes in oceanographic studies and paleo: temperatures, E. Tongiorgi, Ed., Pisa, Italy, 9-130.

Dansgaard W., 1964 : Stable isotopes in precipitation. Tellus, 16, 436-468.

Darling W. G., 2004 : Hydrological factors in the interpretation of stable isotopic proxy data present and past: A European perspective. Quaternary Sci. Rev., $23,743-770$.

Daux V., J. L. Edouard, V. Masson-Delmotte, M. Stievenard, G. Hoffmann, M. Pierre, O. Mestre, P. A. Danis et F. Guibal, 2011 : Ring width, and carbon and oxy- gen isotopic composition of the cellulose in Larix decidua as climatic proxies: A case study in the French Alps. Earth Planet. Sci. Lett., 309, 221-233.

: Dongmann G., H. W. Nürnberg, H., Förstel et K. Wagener, 1974 : On the enrichment of $\mathrm{H}_{2}{ }^{18} \mathrm{O}$ in the leaves of transpiring plants. Radiat. Environ. Biophys., $11,41-52$.

: Etien N., V. Daux, V. Masson-Delmotte, M. Stievenard, V. Bernard, S. Durost, M. T. Guillemin, 0. Mestre et M. Pierre, 2008 : A bi-proxy reconstruction of : Fontainebleau (France) growing season temperature from A.D. 1596 to 2000. Clim. Past, 4, 91-106.

: Etien N., V. Daux, M. T. Guillemin, V. Masson-Delmotte, M. Stievenard, N. Breda, T. Boettger, M. Haupt, O. Mestre et P. P. Perraud, 2009 : Summer maximum - temperature in northern France over the past century: Instrumental data versus multiple proxies (tree-ring isotopes, grape harvest dates and forest fires). Clim. Change, 94, : 429-456.

: Farquhar G. D., M. H. O'Leavy et J. A. Berry, 1982 : On the relationship between carbon isotope discrimination and intercellular carbon dioxide concentration in leaves. : Australian J. Plant Physiol., 9, 121-137.

Francey R. J. et G. D. Farquhar, 1982 : An explanation of 13C/12C variations in tree rings. Nature, 297, 28-31.

: Fritts H. C., G. R. Lofgren et G. A. Gordon, 1979 : Variations in climate since 1602 as reconstructed from tree rings. Quat. Res., 12, 18-46.

Gergis J. L. et A. M. Fowler, 2009 : A history of ENSO events since AD 1525: Implications for future climate change. Clim. Change, 92, 343-387.

: Hilasvuori E., F. Berninger, E. Sonninen, H. Tuomenvirta et H. Jungner, 2009 : Stability of climate signal in carbon and oxygen isotope records and ring width from - Scots pine (Pinus sylvestris L.) in Finland. Journal of Quaternary Science, 24, 469-480.

- Hughes M. K., F. H. Schweingrubber, D. Cartwright et P. M. Kelly, 1984 : July-August temperature at Edinburg between 1720 and 1975 from tree-ring density and : width data. Nature, 308, 341-344.

- IPCC, 2001 : Climate Change 2001a: The scientific basis. Dans : Contribution of Working Group I in The Third Assessment Report of the Intergovernmental Panel on Climate : Change, J. T. Houghton, Y. Ding, D. J. Griggs, M. Noguer, P. J. van der Linden, X. Dai, K. Maskell, and C. A. Johnson, Eds., Cambridge University Press, Cambridge, 881 p.

: Nicault A., Y. Bégin et J. Guiot, 2010 : Standardisation des séries dendrochronologiques. Dans : La dendroécologie, principes, méthodes et applications, S. Payette and - L. Fillion, Eds., Les Presses de I'Université Laval, Québec, 199-227.

- Quinn W. H. et V. T. Neal, 1992 : The Historical record of El Niño events. Dans : Climate since AD 1500, R. S. Bradley and P. D. Jones, Eds., Routledge, London, $623-648$.

- Roden J. S., G., Lin et J. R. Ehleringer, 2000 : A mechanistic model for interpretation of hydrogen and oxygen isotope ratios in tree-ring cellulose. Geochimica et : Cosmochimica Acta, 64, 21-35.

Savard M. M., C. Bégin, J. Marion, D. Arseneault et Y. Begin, 2012 : Evaluating the integrity of $\mathrm{C}$ and 0 isotopes in sub-fossil wood from boreal lakes. Palaeogeogr. Palaeoclim. Palaeoecol., 348-349, 21-31.

: Schweingrubber F. H., O. U. Braeker et E. Schaer, 1987 : Temperature information from a European dendroclimatological sampling network. Dendrochronologia, 5, 9-30.

Schweingruber F. H. et K. R. Briffa, 1996 : Tree-ring density networks for climate reconstruction. Dans : Climatic variations and forcing mechanisms of the last 2000 years, P. D. Jones, R. S. Bradley, and J. Jouzel, Eds., Springer-Verlag, Berlin, Heidelberg, 44-66.

Sidorova 0. V., M. Saurer, V. S. Myglan, A. Eichler, M. Schwikowski, A. V. Kirdyanov, M. V. Bryukhanova, 0. V. Gerasimova, I. A. Kalugin, A. V. Daryin et R. T. W. Siegwolf, 2010 : A multi-proxy approach for revealing recent climatic changes in the Russian Altaï. Clim. Dyn., 38, 175-188.

: Treydte K., G. H. Schleser, G. Helle, D. C. Frank, M. Winiger, G. H, Haug et J. Esper, 2006 : The twentieth century was the wettest period in northern Pakistan over : the past millenium. Nature, 440, 1179-1182.

: Wershaw R. L., I. Friedlan, S. J. Heller et P. A. Franck, 1966 : Hydrogen isotope fractionation in water passing through trees. Dans : Advances in organic chemistry, - Third international congress, G. D. Hobson, Ed., Pergamon, New York, 55-67.

- Yakir D., 1992 : Variations in the natural abundance of oxygen-18 and deuterium in plant carbohydrates. Plant, Cell and Environment, 15, 1005-1020. 